\title{
Jesús Zárate Moreno, trayectoria vital: 1915-1967
}

Jesús Zárate Moreno, life story: 1915-1967

\section{Lunay Estela Figueroa Arias}

Estudiante de Literatura Universidad Autónoma de Bucaramanga (UNAB)

lfigueroa2@unab.edu.co

Artículo recibido el 16 de octubre del 2015 Aprobado el 26 de octubre del $2015 \underline{1}$

\section{Resumen}

Jesús Zárate Moreno (Málaga 1915 - Bogotá 1967), periodista, diplomático y escritor colombiano, autor de varias colecciones de cuentos, incluyendo Un zapato en el jardín (1948), No todo es así (1948), El viento en el rostro (1953), El día de mi muerte (1955) y gran cantidad de artículos y ensayos periodísticos; Y algunas obras publicadas póstumamente: tres piezas de teatro El único habitante, Automóvil en una noche de luna y Cuando pregunten por nosotros en 2003 y dos novelas La cárcel (1972) y El cartero (1973). La cárcel, con la cual ganó el Premio Planeta 1972 en España, es su obra más conocida. Jesús Zárate quien fue un brillante diplomático y ha sido considerado uno de los grandes escritores colombianos, es hoy es uno de los hombres más olvidados de nuestra geografía colombiana.

Palabras Clave: Jesús Zárate Moreno, escritor colombiano, La cárcel, Premio Planeta 1972.

\section{Abstract}

Jesús Zárate Moreno (Málaga 1915 - Bogotá 1967), Colombian diplomat, journalist, essayist and fiction writer, author of several collections of short stories, including Un zapato en el jardín (1948), No todo es así (1948), El viento en el rostro (1953), El día de mi muerte (1955) and numerous essays and newspaper articles; 
and some works posthumously published, three plays El único habitante, Automóvil en una noche de luna and Cuando pregunten por nosotros in 2003 and two novels La cárcel (1972) and El cartero (1973). La cárcel, which won the 1972 Premio Planeta in Spain, is his most famous work. Jesús Zárate, who was a brilliant diplomat and has been considered one of the great Colombian writers, is now one of the most forgotten men of our Colombian geography.

Key words: Jesús Zárate Moreno, Colombian writer, Jail, Premio Planeta 1972.

\section{Introducción}

Los datos presentados en este artículo hacen parte de los resultados de investigación que se adelantan sobre la novela La cárcel de Jesús Zárate Moreno, uno de los autores más representativos de Santander en el siglo XX, quien alcanzó un vasto reconocimiento internacional con el Premio Planeta 1972. El diagnóstico inicial de la investigación dio cuenta de la escasa información disponible sobre la trayectoria vital del autor de la novela y durante el proceso surge la necesidad de organizar y documentar retazos de información biográfica obtenidos de diversas fuentes, necesarios para entender y valorar la importancia literaria del autor y su obra.

Escritor y obra son grandes olvidados de los siglos, corriendo el riesgo de desaparecer a falta de difusión y de estudios que promuevan su conocimiento, discusión y crítica. Se trata, sin duda de una situación que evidencia la necesidad académica y sociocultural de este trabajo que procura valorar la importancia literaria del autor y de su obra, y de esa forma contribuir a su difusión, discusión, promoción, nuevos estudios y su reincorporación al patrimonio literario y cultural de la región y del país.

\section{Metodología}

Los procedimientos que se realizaron para alcanzar los objetivos de la investigación, pertenecen al campo predominantemente cualitativo, y se ciñeron a una estrategia metodológica que abarcó varias etapas: La primera, de ubicación de fuentes primarias y secundarias, apuntando a recopilar la mayor parte de la producción literaria del escritor Jesús Zárate Moreno, constituida por cuentos, obras de teatro y dos novelas La cárcel y El cartero, publicadas póstumamente. En esta etapa además, se recopilaron archivos de artículos y ensayos publicados por el propio autor en periódicos y revistas de la época; adicionalmente, se consultaron escritos de distintos autores sobre la vida de Zárate Moreno y su obra.

La etapa más creativa y por tanto más cualitativa comprendió el análisis e interpretación de los contenidos de la obra para dar respuesta al problema de conocimiento y valoración planteado en el proyecto. 
Esta parte, permitió precisar las conclusiones y sugerencias o recomendaciones tendientes a la difusión del conocimiento de la vida y valor de la obra de Jesús Zárate Moreno que cierran el trabajo.

\section{Resultados}

En Málaga una ciudad de veredas, páramos y ríos, de paisaje accidentado y desafiante, retrato del carácter apacible y batallador de sus habitantes, nació el 15 de septiembre de 1915, el escritor colombiano Jesús Zárate Moreno. Hijo menor de una familia numerosa y de costumbres cristianas, sus padres fueron Isidoro Zárate y Lucía Moreno y sus demás hermanos Saúl, Agustín, Isidoro, Ovidio, Álvaro, Alfredo, Lucía y Rebeca. Carlos Mauricio Serrano, un estudioso de la obra Zárate y familiar del escritor, en su trabajo titulado Jesús Zárate Moreno, entre los mejores del siglo, rememora algunos pasajes de la niñez del autor cobijados por los rumores y el paisaje del campo:

A continuación se presentan cada uno de los talleres clasificados en cada grupo y registrando una descripción de los mismos, el propósito, el desarrollo de actividades y los comentarios o anécdotas que se creyeron relevantes.

En conversaciones con sus primos, hermanos y amigos, evocaban una niñez activa y alegre, con sus viajes semanales a Buenavista, la vereda donde estaba la finca de los abuelos maternos. La vida era sencilla y plácida. Recordaban los caminos, las quebradas, los potreros, los trigales, los árboles y la belleza de las flores y las frutas, que a veces crecían silvestres como los cartuchos, lulos y moras. (Serrano, 2003)

Jesús Zárate Moreno, estudió el bachillerato en el colegio San Jerónimo de su ciudad natal. Serrano cuenta que en las reuniones de amigos y familiares era frecuente evocar historias propias y ajenas de la vida provinciana: "recordaban la gente, los campesinos recios, honrados, hospitalarios y el cura, el médico, el boticario, el tendero, el funcionario de provincia, las muchachas, en fin todos los caracteres que conoció de niño y que tan acertadamente dibujó en sus cuentos" (Serrano, 2003).

Eran las historias cotidianas de los hombres de campo y de las pequeñas ciudades de provincia, que conjugan inocencia y maldad, humor y absurdo, las que fueron alimentando la imaginación del joven Zárate. El crítico y ensayista James J. Alstrum (1982), en el prólogo de la edición póstuma de relatos de nuestro autor No todo es así, nos ofrece esta mirada: "El mundo ficticio que Zárate evocó con acierto, tiene el campo santandereano como escenario. Allí se presentan los absurdos y tragicomedias cotidianas del ciudadano provinciano con plena verosimilitud y la añoranza tierna de recuerdos imborrables" (p. 8).

Por su parte, Hernando Pardo Ordoñez, escritor malagueño y amigo de Zárate, cuenta en Crónicas y 
Anécdotas (1997) algunas de esas historias del diario acontecer sencillo y misterioso de nuestros pueblos: casos como lo sucedido a un famoso médico que llegó de visita por tres días e impresionado gratamente por el sabor del aguardiente pichón, fabricado en Málaga, terminó quedándose hasta ser sorprendido por la muerte ya sin hígado (p. 187); o de la primera cirugía de cataratas hecha en Colombia en 1926, realizada a Bernabé, un aguatero ciego, "es como estar en el reino de los cielos", dijo al ver el color de la flores después de cincuenta años (p. 499); la historia del loco Meléndez quien soñó realizar un espectáculo teatral que incluía el incendio del pueblo con el dinero recaudado a los asistentes reconstruiría la Málaga moderna y soñada (p. 247); o cómo la de ese mismo loco, con las manos en la garganta de un hombre que evadía impune la justicia, logra la confesión frente a todos los asistentes de la iglesia, de ser el asesino del avaro rico del pueblo (p. 250); y así, más historias de promeseros, estafadores, cerveceros, ladrones, agiotistas, avaros, etc.

Son historias del trasegar de provincia, en las que se conjuga el humor, el absurdo, la ironía con la cotidianeidad y el espíritu arbitrario que constituyeron la materia prima para la obra cuentística de Jesús Zárate. El reconocido ensayista y crítico literario Hernando Téllez (1949) en su análisis No Todo es Así: los temas de la provincia, sobre el libro de cuentos del mismo nombre de nuestro autor, enfatiza el acertado manejo del desarrollo del tema provincial como mérito literario de la obra, y nos revela su impresión a partir del libro del testimonio vital de Zárate:

Lo mejor de tu experiencia vital y de tu experiencia intelectual, viene de la provincia, de la comunicación indestructible que, en la infancia, en la primera juventud, tuviste con las cosas, con las criaturas, con los animales, con la atmósfera de unas breñas colombianas y de unos pueblos solitarios y tristes. (p. 4)

Dentro de la novela La cárcel (1972), estos relatos de sentido provinciano, abundan con sutil toque de humor irónico. El siguiente pasaje es buen ejemplo, que diluye la delicada franja entre la razón y la locura y deriva intencionada hacia la filosofía:

-En Málaga, donde yo nací, conocí una vez a un filósofo. Es el único filósofo que he visto cara a cara. Se llamaba Donato Cruzado. Poco antes de que lo llevaran al manicomio le oí una frase que es lo mejor que se ha dicho sobre la vida. Donato Cruzado dijo: "Si yo no fuera loco, me volvería loco". (p. 284)

Contrario al deseo de sus padres, a la edad de 15 años, el hijo menor de la familia Zárate Moreno, se traslada a Bucaramanga. Alstrum (1982) cuenta: “sus padres querían que Jesús se quedara en la finca 
dedicado a la vida pastoril" (p. 8). Bucaramanga, capital del departamento de Santander, acoge a los hombres de provincia que convergen a ella atraídos por el deseo de trascender en la empresa, en la política o en las letras. Jesús Zárate se inscribe en el colegio de San Pedro Claver, para terminar sus estudios de bachillerato. En uno de sus primeros cuentos: Las bodas del bachiller, evoca este tradicional colegio sentado en el parque aledaño, punto de encuentro de amigos o de repaso a los exámenes finales:

Aquel día terminaban los exámenes finales. Gabriel Pinto y Mario Aldana, alumnos de sexto año de literatura, se encontraban estudiando en el parque. Desde los bancos de madera donde estaban sentados veíase el viejo edificio del Colegio de San Pedro Claver. El parque tenía el aspecto de un solar abandonado. Los árboles extendían sus ramajes con insolencia silvestre sobre los senderos del paseo, y las flores languidecían en los prados, tostados bajo el sol de noviembre (...) barnizado de blanco y amarillo. En los muros exteriores se veían las inmensas vetas negras formadas por el hollín que arrastraban las lluvias. (Zárate, 1982, p.105)

Zárate Moreno, pronto obtiene su primer empleo como periodista para la revista Aire Libre de Gustavo Puyana. Hacia el año 1936, se vincula con Vanguardia Liberal, principal periódico local, en ese momento bajo la dirección de Tomás Vargas Osorio, destacado poeta santandereano del grupo Piedra y Cielo. A Vargas Osorio lo unió una pasión mutua por el periodismo y la literatura y una cercana amistad que queda demostrada en una carta de 1939, donde le confiaba a Zárate Moreno, algunas intimidades de su último proyecto literario:

Yo estoy trabajando seguido en un libro nuevo titulado "La Familia de la Angustia". Hasta ahora llevo la mitad más o menos, y estoy satisfecho de ese trabajo. Es un análisis anticonceptualista, antiliterario, intuitivo de mis autores preferidos: Nietzsche, Dostoievski, Unamuno (...). Me está resultando un ataque violento al racionalismo, al humanismo, en fin a la cultura occidental. (Zárate, 1942, p.2ª

Sus habilidades de periodista y escritor, rápidamente son reconocidas por el propietario del periódico Vanguardia Liberal Alejandro Galvis Galvis, quien al poco tiempo le dio la oportunidad de convertirse en jefe de redacción de su diario (Alstrum 1982: 8). En 1940, inicia su etapa periodística en diarios de circulación nacional. Roberto García Peña (1967), recuerda que "siendo estudiante en Bucaramanga, desempeñó con eficacia y brillo singulares la corresponsalía de El Tiempo, vinculándose así, desde entonces, a esta casa espiritual que le contara entre sus mejores amigos" (p. 4).

Jesús Zárate Moreno, como la mayoría de los intelectuales y hombres de negocios, no pudo sustraerse a 
los cánones tradicionales de la época y orbitó alrededor de la política. En su escrito La vida y obra de un grande escritor santandereano: Tomás Vargas Osorio (Zárate, 1942) manifiesta preocupación particular sobre el dilema del intelectual frente a las camarillas de la política:

El periodista desemboca en la política. Lo mismo el escritor. Esto explica la languidez de nuestra literatura. Las luchas de partido aniquilan en Colombia las mejores inclinaciones literarias. El mal no se corregirá, mientras los espíritus realmente aptos para hacer brillar la cultura nacional tengan que seguir hipotecando su inteligencia para comer. (p. 2) ).

\section{Conclusiones}

Carlos Mauricio Serrano, en su texto: Jesús Zárate Moreno, entre los mejores del siglo, recuerda la época de intenso trabajo de campaña como representante a la Cámara por la provincia de García Rovira, en Santander, de la que Zárate regresa derrotado, luego de tres meses de gira y discurso. Hablando del tema con su amigo Gustavo Cote Uribe, le dice a éste: "entre la política sin amor y Alicia, me quedo con Alicia definitivamente" (Serrano, 2003). Flor Romero de Nohra, por su parte, en su escrito Gloria Literaria después de muerto (Romero, 1972) cuenta: "Él era de Málaga, y ella es de Bucaramanga. Se casaron cuando Alicia tenía 18 años, y fue su secretaria, quien le ayudaba en la corrección de las obras, y a pasarlas en limpio”. Fruto del matrimonio con Alicia Rey nacieron sus tres hijos: Néstor Augusto, Eduardo y Alicia.

El año 1941, trajo motivos de profundo pesar a Jesús Zárate, comenta Serrano (2003): “en este año murió su madre doña Lucía Moreno de Zárate, hecho que le afectó enormemente” y el 21 de diciembre fallece su amigo cercano, el ilustre escritor Tomás Vargas Osorio. Le corresponde a Jesús Zárate siendo corresponsal en Santander para El Tiempo, reportar con inmenso dolor al día siguiente la dolorosa noticia: “(...)su muerte constituye una pérdida sensible por mil motivos para las letras patrias, y para el periodismo (...), del cual fue Vargas Osorio una de sus más brillantes unidades" (Zárate, 1941: 1). En una hermosa semblanza que publica posteriormente en El tiempo, sobre la vida y obra del poeta, Zárate (1942) recuerda la dimensión poética por la que fue reconocido el piedracielista: "Una vez cantó como Dante, lo que está más allá de la agonía. Cantó el retorno de la muerte" (1 de febrero 1942: 2).

Al recordar a Vargas Osorio, reflexiona sobre sus propias aficiones: "Los dos vicios de la inteligencia son el periodismo y la política. Esta por lo que tiene en sí de pervertido. Aquél porque casi nunca se sirve con desinterés y probidad intelectual”. Pero, revela García Peña (1967) que el desencanto por la política le duraría poco tiempo, retorna al ruedo político para trabajar como subsecretario de gobierno de Santander, "posición de la cual fue llamado por el doctor Gabriel Turbay para que lo acompañara como su secretario particular" (p. 26). Al prestigioso diplomático y dirigente liberal, lo había conocido mientras trabajaba en el diario 
Vanguardia Liberal y desde entonces se hicieron grandes amigos. (Alstrum, 1982).

El año 1943 marca el inicio de su brillante carrera diplomática. Alicia Rey dice: "nos vinimos a Bogotá, porque Jesús fue nombrado jefe de correspondencia del Ministerio de Relaciones Exteriores” (Romero, 1972). Un nombramiento que registra complacido su amigo Roberto García Peña (1943) en el periódico El Tiempo: “llegó ayer a Bogotá, procedente de Bucaramanga y con el propósito de tomar posesión de un alto cargo en el Ministerio de Relaciones Exteriores" resaltando sus cualidades personales e intelectuales, como: "una de las figuras jóvenes más gallardas de la inteligencia santandereana. Escritor de magnífica prosa y polemista sagaz y combativo, Zárate Moreno no obstante su juventud, ocupa ya un puesto de prestigio en el periodismo de Santander" (p. 5).

Por ésta época, Jesús Zárate Moreno escribe sus primeros cuentos. El investigador, Eduardo Pachón Padilla (1959), en su Antología del Cuento Colombiano, incluye su cuento La cabra de Nubia (1949) y en la nota biográfica-crítica sobre su autor, afirma: "se inició en el género cuentístico a principios de 1944, con su trabajo: Fin del Mundo, publicado en el suplemento de El Tiempo de Bogotá" (p. 287); reincorporándose al periódico para colaborar con notas, ensayos y cuentos de publicación especial en el Suplemento Literario de los domingos. Pachón (1959) dice: “desde entonces, su labor literaria ha sido difundida en las más importantes revistas y periódicos del país, y algunos de sus cuentos han sido vertidos al inglés” (p. 287).

En el año 1946 continúa su labor en la cancillería y el servicio exterior, "con una breve interrupción durante el tiempo en que fue nuevamente secretario particular del doctor Gabriel Turbay, durante la campaña presidencial adelantada por el insigne hombre público", recuerda Roberto García Peña (1967), y se dedicaría de tiempo completo a trabajar para el candidato oficial del partido liberal. Jorge Padilla (1986), compañero de trabajo de Zárate, recuerda momentos de campaña:

Estamos en 1946. Acompañamos a Gabriel Turbay, candidato a la Presidencia de la República, en la fragorosa campaña que desembocaría en la pérdida del poder. Somos una comitiva de intelectuales y políticos en borrador. César Ordoñez Quintero, Jesús Zárate Moreno, Luis Enrique Figueroa, Augusto Espinosa Valderrama y yo, desguarambilados, las camisas desechas y los cabellos revueltos, intentamos arreglar la república y garlamos con ligereza de la juventud, sobre todo lo humano y lo divino (p. 4).

Gabriel Turbay, enfrenta en intención y deseo a su copartidario el caudillo Jorge Eliécer Gaitán, luego de una división de partido que causa la derrota del liberalismo el 6 de mayo de 1946 y facilita la victoria del partido conservador unido en torno al candidato Mariano Ospina Pérez, alcanzando por fin las mieles de 
poder, del que adolecía desde 1930. Alstrum (1982) refiere al respecto: "Colombia ya se encontraba en vísperas de una de las épocas más sombrías de su historia cuando Turbay, el derrotado candidato presidencial le gestionó a su ex secretario un cargo diplomático en el exterior" (p. 9). A partir de 1946 inicia su fructífera labor diplomática. Jesús Zárate es nombrado Primer Secretario de la Legación colombiana en Madrid y en octubre asume además, como Cónsul General Encargado de Colombia en España, con sede en Barcelona. El día 10 de octubre, el diario La Vanguardia Española (1946), reseña uno de los últimos encuentros de amigos, en la atención ofrecida por el cónsul Jesús Zárate al ilustre ex candidato Gabriel Turbay en España:

En avión llegó ayer por la tarde de Madrid, el ilustre jurisconsulto y exministro de negocios extranjeros de Colombia, doctor Gabriel Turbay, que realiza un viaje de turismo y observación por Europa (...). Antes de reanudar su viaje con dirección a Francia, permanecerá unos días en esta ciudad con el objeto de conocer nuestras instituciones culturales (...). Fue recibido por el cónsul de Colombia, don Jesús Zárate. (p. 10)

La realidad europea luce muy diferente de la imagen que le habían vendido, pocos meses después de su llegada reflexiona sobre ella, con el crítico más radical de la decadencia española, el escritor Pío Baroja, a quien visitó varias veces en su casa de Madrid. Jesús Zárate plasmará algunos años después, aquellas impresiones recibidas de primera mano, en su escrito Pío Baroja: Hombre humilde y errante, en donde dice:

A Baroja le preocupa mucho la decadencia hispánica, y para explicarla utiliza los mismos recursos estéticos que aprovecha en sus novelas. El absurdo -siempre el absurdo- es su mejor herramienta filosófica. Baroja analiza la religión, la raza, la lengua, la música y las costumbres españolas y llega a una conclusión desconsoladora (Zárate, 1949, p. 4).

La mirada de Baroja libre de hipocresía, realista y profunda sobre la devastada España sumada a sus agudas observaciones en los "frecuentes asomos a los países circunvecinos le convencieron de que Europa, la Europa de ahora, no eran como se la habían pintado", comenta Roberto García Peña (1947) y Zárate, revierte ese desencanto en un lúcido ensayo El hombre de Europa que fue publicado en la revista América en el mes de junio de 1947.

Mientras Zárate vivía en España, aparece su primer libro de relatos Un zapato en el jardín (1948), compuesto por una serie de trece cuentos. Publicado en la ciudad de Madrid y con el auspicio de la editorial 
Afrodisio Aguado, quien reseña en el libro:

La experiencia de las rotativas le dio la rapidez y la certeza de enfoque para los hechos y los hombres que campean en las páginas de "Un zapato en el Jardín". Dióle además el ligero tinte de humor que irisa la prosa fácil y transparente. Y, al lado de esto, las reiteradas lecturas, la copiosa información de las literaturas inglesa y norteamericana contemporáneas, el talento seguro de quien domina por igual el asunto y los instrumentos de expresión, todo confluye a hacer de Zárate Moreno uno de los primeros cuentistas colombianos. (Zárate, p. 1).

Jesús Zárate Moreno publica en el mismo año en Colombia, con la editorial Imprenta de Bucaramanga, un segundo libro No todo es así (1948), que incluye una colección de diez cuentos. Zárate (1948) explica el origen del título, en la nota que a manera de prólogo introduce el libro (fechada en Bogotá, febrero de 1946):

Con varios amigos solíamos discutir todas las tardes sobre política y literatura, en una tarraza de la Avenida de la República. (...) Un día Carlos Prados me dijo: “Tus cuentos pueden ser buenos o malos, pero son cuentos. Tienen como tales una característica esencial, basada en la discreta combinación de lo vulgar y de lo misterioso. Al leerlos cualquiera diría que nuestra pequeña historia carece de esas sorpresas, y que en la vida NO TODO ES ASI. (Zárate, p.25)

A su regreso a Colombia, Jesús Zárate es nombrado como subdirector del departamento diplomático de la cancillería entre los años 1948 y 1949 y sigue escribiendo con regularidad sus informes especiales para el periódico El Tiempo. Hernando Téllez (1949), en carta dirigida a Jesús Zárate, publicada en el suplemento literario de El Tiempo, en referencia a la lectura de su primer libro de cuentos, le expresa una clara admiración por su estilo literario y por la universalidad de los temas:

Hay en tu libro, universalmente legible, una recuperación espléndida de los temas de provincia, tan desvaídos, tan débiles, tan insubstanciales en otras manos (...). Nada importa que un día te encuentres en París o en Barcelona, en Madrid o en Londres o que, como ahora, veas discurrir tus días en los salones del Ministerio de Relaciones Exteriores de Colombia (...). Tu libro demuestra que lo mejor de tu experiencia vital y de tu experiencia intelectual, viene de la provincia, de la comunicación indestructible que, en la infancia, en la primera juventud, tuviste con las cosas, con las 
criaturas, con la atmósfera de unas breñas colombianas y de unos pueblos solitarios y tristes, en donde el tedio es la verdadera dimensión de la vida. (p. $4^{\mathrm{a}}$ )

Jesús Zárate Moreno se desempeña como director de información y prensa de la cancillería en 1949. Este mismo año se vincula con el periódico El Espectador y pasa a ser uno de sus asiduos colaboradores con notas para el Magazín Dominical con el título: El pabellón del Reposo usando el seudónimo, Zalacaín (Romero, 1972). Notas basadas en historias tomadas de la vida real contadas con un tratamiento literario depurado, en forma de relatos cortos, que fueron publicados semanalmente y de forma continua hasta el año 1954. La periodista y escritora Flor Romero de Nohra (1972), cuenta de aquella época:

Lo recuerdo llegando al edificio de El Espectador en la avenida Jiménez con una compostura de lord, paraguas, y guantes de cuero, una sonrisa medio burlona, y las cuartillas del Pabellón del Reposo en la mano "que yo quiero corregir cuando esté ya levantado, porque a veces me hacen unas empasteladas...”.

En el completo análisis del crítico y escritor Germán Arciniegas Gatopardo a la colombiana (1982), sobre La cárcel la señala como "una de las mejores novelas castellanas" y nos acerca a la elegancia de su estilo, al que califica de "limpieza inmaculada". El crítico describe a Jesús Zárate con estas palabras:

A los cinco años el muerto se escapa -iyo soy un hombre libre!- y con el mismo ingenio, la misma vivacidad - ¡las chispas de sus ojillos zahoríes!- retorna para contarnos las historias de la cárcel. Otra vez la frescura de su risa que nunca fue estrepitosa sino inteligentísima (...). Zárate, príncipe de las montañas de Colombia, comunicativo, era de una cara aceitunada gorda; casi le cerraba los ojos cuando reía. Y su corbatín de mariposa, rúbrica de travesura provinciana. (p. 298)

Durante 1950 hasta el año 1953, Jesús Zárate trabaja como jefe de la sección de Europa, África y Oriente del departamento diplomático de la Cancillería. En este último año, aparece su tercer libro de cuentos El viento en el rostro, relatos de Zalacaín (1953), publicado en Bogotá con Ediciones Espiral y es una selección de las cincuenta de las mejores crónicas periodísticas escritas para el magazín dominical de El Espectador. La revista trimestral de la Universidad Nacional de Colombia (1953), reseña este libro como uno de los mejores 
publicados en los últimos años:

Estos son esencialmente objetivos, periodísticos y las obras que comprende el libro de Zárate, muestran no obstante su brevedad, altas cualidades arquitectónicas de composición. Por otra parte, se encuentra en estos cuentos una gran variedad de asuntos, hasta el punto que no hay nexos entre uno y otro, sino es el estilo claro y preciso del autor, que se une a las cualidades ya anotadas para hacer de éste uno de los mejores libros que se han publicado en Colombia en los últimos años. (p. 212)

Jesús Zárate Moreno es promovido director de Política Exterior y del Departamento Diplomático entre 1953 a 1954. Continúa con sus actividades diplomáticas como consejero de la Embajada de Colombia en México de 1954 a 1955. En éste último año, Jesús Zárate publica su cuarto libro de cuentos, compuesto por trece relatos, que llamó El día de mi muerte, con el sello de la Editorial Iqueima de Bogotá, con el auspicio del Ministerio de Educación Nacional. James Alstrum (1982) califica el libro de Zárate, como:

Su colección más lograda de cuentos, revela que Zárate había tomado en serio los conceptos de Téllez. Las historias santandereanas encontradas en este libro, igual que los relatos antioqueños de Tomás Carrasquilla (1858-1940), trascienden el costumbrismo cursi y alcanzan un verdadero interés universal” (p.11).

Algunos cuentos son testimoniales del ambiente de violencia posterior a los hechos lamentables del 9 de abril de 1948, que marcaron en nuestro país un viraje temático de la literatura del costumbrismo hacia la literatura de violencia. Sebastián Pineda Buitrago (2012) en Breve historia de la narrativa colombiana. Siglo XVI-XX (2012), señala el tratamiento del tema en los cuentos de Jesús Zárate, y a su vez reconoce que "no se trata de la violencia macabra o truculenta, sino de una muy estética, cargada de matices psicológicos” (p. 239).

La siguiente escala de Jesús Zárate en la diplomacia es como consejero de la Delegación de Colombia ante la OEA, cargo que desempeñará hasta 1956. En el periodo de 1956 a 1958, Jesús Zárate trabaja como asesor consejero de la delegación de Colombia ante la ONU, país elegido por tercera vez como miembro no permanente del consejo de seguridad; después en la categoría de subsecretario del Servicio Exterior en disponibilidad en la cancillería, durante los años 1958 a 1959; continuando su ascenso diplomático a subsecretario de asuntos diplomáticos de la cancillería, entre 1959 a 1960.

Jesús Zárate, es nombrado director del Ministerio de Relaciones Exteriores y Delegado alterno de Colombia como Ministro plenipotenciario ante la XVI Asamblea General de las Naciones Unidas, durante el 
periodo 1960 a 1961.

Luego de su brillante desempeño en la diplomacia, a Jesús Zárate Moreno se le es confiada la difícil tarea de manejar la primera Embajada de Colombia en Cuba, en época de transición del gobierno de la dictadura de Batista al régimen revolucionario de Castro. Roberto García Peña (1967), destaca la sobresaliente actuación de nuestro embajador, al afirmar: "se hizo brillante, porque su tarea estuvo en todo momento señalada no solo por el decoro de su conducta, sino por la devoción patriótica con que adelantó las misiones que le fueron encomendadas." De aquella experiencia de alta tensión en el manejo de las relaciones diplomáticas con la Habana, nació la idea de escribir su primera novela, revela su esposa Alicia:

Recuerdo que Jesús me contaba de los cuatro meses que pasó encerrado en un hotel, en donde funcionaba por ese entonces la Embajada; como tenía asilados políticos las puertas del hotel fueron custodiadas por barbudos; a lo que mi marido le tenía pánico era a que se le disparara una ametralladora a alguno de los novatos centinelas. Por entonces fue que concibió la idea de escribir "La cárcel", una especie de diario de 230 páginas, que el dejó con tres copias mecanografiadas y con el seudónimo de Pablo Lepanto. (Romero, 1972)

Posteriormente, Jesús Zárate es nombrado embajador de Colombia en la República Dominicana en 1962, un año más tarde "obraría con firmeza e integridad y extraordinaria habilidad" en ardua labor de intermediador por solicitud del gobierno de la República Dominicana a su embajada en Puerto Príncipe, de encargarse de la representación de los intereses dominicanos en Haití y de las personas asiladas en la embajada dominicana, en la época crisis generada por la ruptura de las relaciones entre los dos países hermanos. (García, 1967).

Con igual gallardía, Jesús Zárate Moreno supo sortear la situación durante los difíciles acontecimientos de la revolución en 1965, que condujeron a una violenta guerra civil en República Dominicana. El periodista Carlos J. Villar Borda, quien permaneció durante seis meses en la isla en la época de la invasión estadounidense a República Dominicana, posterior al asesinato del dictador Trujillo, recuerda algunas visitas a la casa de su amigo Jesús Zárate:

El embajador era Jesús Zárate Moreno, un escritor, novelista y cuentista muy amigo mío, que se solía reunir a la hora de comer, con otros embajadores latinoamericanos y hombres de letras latinoamericanos dominicanos que podían o se atrevían a salir de su vecindad con la embajada. (...) En estas veladas nocturnas obtenía y daba información y luego pasábamos a hablar de literatura o de arte. (p. 331)

Al embajador Jesús Zárate, le correspondió realizar allí una encomiable y extraordinaria labor frente a los graves episodios internos presentados durante ese período en República Dominicana, revela García Peña 
(1967):

(...) le tocó vivir, allí, asistido por la fortaleza moral de su incomparable compañera doña Alicia Rey de Zárate, las más amargas horas durante la violenta y sangrienta guerra civil que golpeó a aquel infortunado país y determinó muy duras condiciones de vida a quienes tuvieron que afrontar las consecuencias de la anárquica emergencia. (p. 4)

Zárate Moreno, recibió "los más altos elogios a su conducta" por la encomiable labor como embajador de Colombia en República Dominicana; Al retorno a la democracia, Zárate fue honrado por este gobierno para hablar de su país, demostrando su talento literario en el lúcido ensayo Así es Colombia: Lección en mil palabras (1966).

En la labor diplomática, Zárate Moreno mantuvo siempre la elegancia de lo discreto y su modestia la prestancia de lo auténtico, evoca Roberto García Peña (1967) y quizás, “aquellos días fueron intensos y agotadores y acaso de ellos nació la tremenda enfermedad que había de destruir el vigor de su juventud" (p. 4). Su larga estancia como embajador en Santo Domingo, permitió a Jesús Zárate, escribir su primera novela La cárcel, que permaneció inédita en su maletín hasta cinco años después de su muerte.

La enfermedad había avanzado silenciosamente y durante su permanencia en Santo Domingo se manifestaron los primeros síntomas. "Jesús nunca se quejaba de nada, tenía una salud a toda prueba", recuerda Alicia, su esposa y dice que "cuando empezó la fiebre alta y malestar, pensábamos que era un resfriado, pero cuando pasaron 8 días y no había mejoría”, entonces presintió la gravedad de la enfermedad y pensó en buscar ayuda. Flor Romero (1972), dice que Jesús intentó tranquilizarla apelando a su buen humor: "yo sé que en Bogotá me curo. Lo que pasa es que estoy aburrido en este Caribe con tantos golpes que me han tocado”. En Bogotá fue atendido e intentaron operarlo en el Hospital Militar; los médicos conceptuaron que no había nada qué hacer.

Aferrados a la esperanza, los esposos Zárate deciden viajar a Nueva York. "En el Instituto Park nos dieron alguna esperanza y empezaron el tratamiento de cobalto, pero yo veía que se acababa día a día. El trataba de animarme, de decirme que había que vivir; era una situación dramática, ignorándolo y sabiéndolo ambos”, recuerda su esposa. Jesús Zárate Moreno, mantuvo el buen humor a pesar de la tragedia interior, Alicia cuenta que él odiaba el luto, y que al verla tan preocupada por su enfermedad, un día le dijo: “¿Estamos en verano, por qué no vas y te compras un vestido rojo, que te queda muy bien? Ella lo buscó por todas partes para complacerlo, pero no lo encontró. Los esfuerzos para la recuperarse de sus prolongadas dolencias resultaron inútiles intentos: "parece que fue hace un mes cuando comenzó a adelgazar, a empalidecer, a sentirse mareado por las tardes y a dejar de ser el hombre fuerte y animoso que ella tuvo como compañero por más de 20 años", 
recuerda Alicia. (Romero, 1972).

Con dolor y resignación deciden retornar a Bogotá. Allí, Zárate escribe su segunda novela: El cartero, Cuenta Alicia que "se dedicó por completo a escribir día y noche. 'no quiero que me moleste nadie', nos dijo y se encerró a teclear" una obra llena de suspenso y humor negro, que estructuró en tres meses. Dice además que "él era una persona muy ordenada, y dejó esta obra terminada con correcciones al margen, lo mismo que las dos piezas de teatro". (Romero, 1972)

En 1967, Zárate recibe una última misión en la diplomacia, la embajada de Colombia en Suecia; pero, la enfermedad y la inminencia de su muerte le impidieron cumplir esta tarea. Jesús Zárate Moreno muere en Bogotá, el 12 de diciembre de 1967. Roberto García Peña (1967), amigo y director del periódico El Tiempo, lo despide de este mundo con estas emotivas palabras:

No importa que desde hace meses los suyos y sus amigos, asistiéramos con creciente amargura a la inminencia de su muerte. El que esta haya llegado liberadora de sus sufrimientos para Jesús Zárate Moreno, no disminuye la congoja que nos causa su realidad tremenda. Porque la desaparición de este colombiano ejemplar, de este compañero para siempre inolvidable, se produce cuando muchas cosas podía la vida haberle dado todavía, pues eran muy claros los dones de su espíritu y ellos autorizaban confiar en que mucho debía aguardarse de su inteligencia y de su diligencia, durante más de cinco lustros consagrados al servicio de su patria. (p. 4)

\section{Referencias}

\section{Referencias}

Alstrum, J.J. (1982). "Prólogo”. En: Zárate, J. No todo es así. Bogotá: Instituto Colombiano de Cultura. (pp. 7-20).

Arciniegas, G. (1982). “Epílogo”. En, Zárate, J. No todo es así. Bogotá: Instituto Colombiano de Cultura, (pp. 295-300).

García, R. (1943, 10 de enero). “Cosas del día: Jesús Zárate Moreno”. Periódico El Tiempo. . (1947, junio). “Temas y Autores”. Revista de América. Volumen X (30). . (1967, 13 de diciembre). “Cosas del día: Jesús Zárate Moreno”. Periódico El Tiempo. . (1967, 13 de diciembre). "Falleció ayer el escritor Jesús Zárate Moreno”. Periódico El Tiempo.

Llegada de una destacada personalidad colombiana. (1946, octubre 10). La vanguardia española.

Padilla, J. (1986, 06 de octubre). "Pétalo sobre la sombra de un político”. Periódico El Tiempo.

Pardo, H. (1997). Crónicas y Anécdotas. Bucaramanga: Sic. 
Pachón, E. (1959). Antología del Cuento Colombiano. Bogotá: Editorial A.B.C.

Pineda, S. (2012). Breve historia de la narrativa colombiana. Siglos XVI-XX. Bogotá: Siglo del Hombre Editorial.

Romero de Nohra, F. (1972, 17 de diciembre). “Gloria Literaria Después de Muerto•. Magazín Dominical El Espectador.

Serrano, C. M. (2003, abril). “Jesús Zárate Moreno, entre los mejores del siglo”. Revista Estudio, núm. 331. Academia de Historia de Santander.

Téllez, H. (1949, 27 de marzo). “No todo es así: los temas de provincia”. Suplemento Literario, El Tiempo. Universidad Nacional de Colombia. (1953). Revista trimestral de cultura moderna. Número (17-21).

Villar, C. J. (2004). La pasión del periodismo. Bogotá: Fundación Universidad de Bogotá Jorge Tadeo Lozano.

Zárate, J. (1941, 22 de diciembre). "Falleció ayer Vargas Osorio en Bucaramanga”. Periódico El Tiempo. . (1942, 02 de enero). "La vida y la obra de un grande escritor santandereano: Tomás Vargas Osorio".

Suplemento Literario, El Tiempo.

. (1947, junio). “El hombre de Europa”. Revista América, Volumen X (30). p. 354.

. (1948). Un zapato en el Jardín. Madrid: Afrodisio Aguado.

. (1949, 16 de enero). "Pío Baroja: Hombre humilde y errante”. Suplemento literario, El Tiempo, p. 4.

. (1961). "Carta a secretario de relaciones exteriores de Cuba”. El tiempo.

. (1966). “Así es Colombia: Lección en mil palabras”. Suplemento Literario, El Tiempo.

. (1972). La cárcel. Barcelona: Planeta.

(1982). No todo es así. Bogotá: Instituto Colombiano de Cultura.

. (2003). Tres piezas de teatro. Bogotá: Instituto Caro y Cuervo.

Artículo arbitrado por Claudia Patricia Mantilla Durán. Magíster en Semiótica. Universidad Industrial de Santander. Docente Universidad Autónoma de Bucaramanga.

Citar este artículo como: Figueroa, L. (2015) “Jesús Zárate Moreno, trayectoria vital: 1915-1967”. En:

Revista La Tercera Orilla (15). Bucaramanga: Universidad Autónoma de Bucaramanga.

Artículo arbitrado por Johnattan Farouk Caballero Hernández. Doctorando de la Universidad Autónoma de México (Méx.) y Magíster en Literatura de la Universidad de los Andes (Col.) 